Although this space did not result in any complications, it is the opinion of the surgeon (T.F.) that in hindsight it might have been reasonable to forego the mediastinal dissection. In this case a mediastinoscopy had been performed preoperatively, and avoiding lymphadenectomy would have eliminated the risks associated with this postoperative space. Unlike a primary resection, in which resection of the upper and lower lobes would place the middle lobe at significant risk of torsion in a reoperation, adhesions encountered in the second operation appear to reduce this risk. Iwata and colleagues ${ }^{5}$ reported a similar clinical scenario occurring on the left side, where a recurrent tumor was treated with segmentectomy to avoid completion pneumonectomy. This approach is appealing, because surgical mortality after completion pneumonectomy is reported as $20 \%$ for the right lung and $10 \%$ for the left. ${ }^{3}$

In light of this patient's limited pulmonary reserve, her oxygen requirements after her original lobectomy, and the high mortality associated with completion right pneumonectomy, ${ }^{3}$ we chose to save the middle lobe. We are unable to identify any citation stating that this approach is contraindicated. From many conversations with peers, however, most thoracic surgeons appear to believe that preserving the middle lobe by itself is in fact contraindicated or at least unwise. In the absence of evidence-based citations, reports to prove or disprove this approach are of value.

This single case report does not represent proof that this was the appropriate treatment. It may, however, stimulate interest and lead to further evaluation to support or refute this approach.

\section{References}

1. Adebonojo SA, Moritz DM, Danby CA. The results of modern surgical therapy for multiple primary lung cancers. Chest. 1997;112:693-701.

2. Fabian T, Bryant AS, Mouhlas AL, Federico JA, Cerfolio RJ. Survival after resection of synchronous non-small cell lung cancer. J Thorac Cardiovasc Surg. 2011;142:547-53.

3. Guggino G, Doddoli C, Barlesi F, Acri P, Chetaille B, Thomas P, et al. Completion pneumonectomy in cancer patients: experience with 55 cases. Eur J Cardiothorac Surg. 2004;25:449-55.

4. Angeletti CA, Mussi A, Janni A, Lucchi M, Ribechini A, Chella A, et al. Second primary lung cancer and relapse: treatment and follow-up. Eur J Cardiothorac Surg. 1995;9:607-11.

5. Iwata H, Shirahashi K, Mizuno Y, Matsui M, Yamamoto H, Takemura H. Left lower apical segmentectomy after video-assisted left upper lobectomy for relapse in lung cancer: benefit to avoid completion pneumonectomy. Gen Thorac Cardiovasc Surg. 2013;61:359-61

\title{
Extracorporeal membrane oxygenation as a bridge to surgery for ischemic papillary muscle rupture
}

\author{
Benjamin Obadia, JD, Alexis Théron, MD, Vlad Gariboldi, MD, and Frederic Collart, MD, Marseille, \\ France
}

\section{VICS Video clip is available online.}

For patients in highly unstable condition, a mobile remote cardiac assist unit (MRCAU) was created in our institution to allow implantation of an extracorporeal membrane oxygenation (ECMO) system while the patient is in a peripheral hospital, before refractory multiorgan failure

From the Department of Cardiac Surgery, La Timone Hospital, Marseille, France. Disclosures: Authors have nothing to disclose with regard to commercial support. B.O. and A.T. contributed equally.

Received for publication Dec 9, 2013; revisions received Feb 1, 2014; accepted for publication March 3, 2014; available ahead of print March 26, 2014.

Address for reprints: Alexis Théron, MD, Department of Cardiac Surgery, La Timone Hospital, 264 Rue Saint Pierre, 13385 Marseille Cedex 05, France (E-mail: alexis. theron@ap-hm.fr).

J Thorac Cardiovasc Surg 2014;147:e82-4

$0022-5223 / \$ 36.00$

Copyright (c) 2014 by The American Association for Thoracic Surgery

http://dx.doi.org/10.1016/j.jtcvs.2014.03.003 occurs. ${ }^{1}$ We report the successful treatment of a complete posteromedial papillary muscle rupture complicating an inferior ST-segment elevation myocardial infarction supported by percutaneous ECMO implantation as a bridge to surgery.

\section{CLINICAL SUMMARY}

A 67-year-old man with a history of hypertension called emergency medical services for brutal chest pain and breathlessness. At the emergency unit arrival, he was in acute respiratory failure with cardiogenic shock. He was immediately intubated and brought back to the intensive care unit. At admission, physical examination revealed an unknown $4 / 6$ holosystolic murmur at the apex and significant bilateral rales. Electrocardiography showed an ST-segment elevation in leads II, III, and aVF, suggesting an inferior ST-segment elevation myocardial infarction. Chest radiography revealed an acute pulmonary edema and ruled out left ventricular and left atrial dilatation (Figure 1). Transthoracic echocardiography revealed 

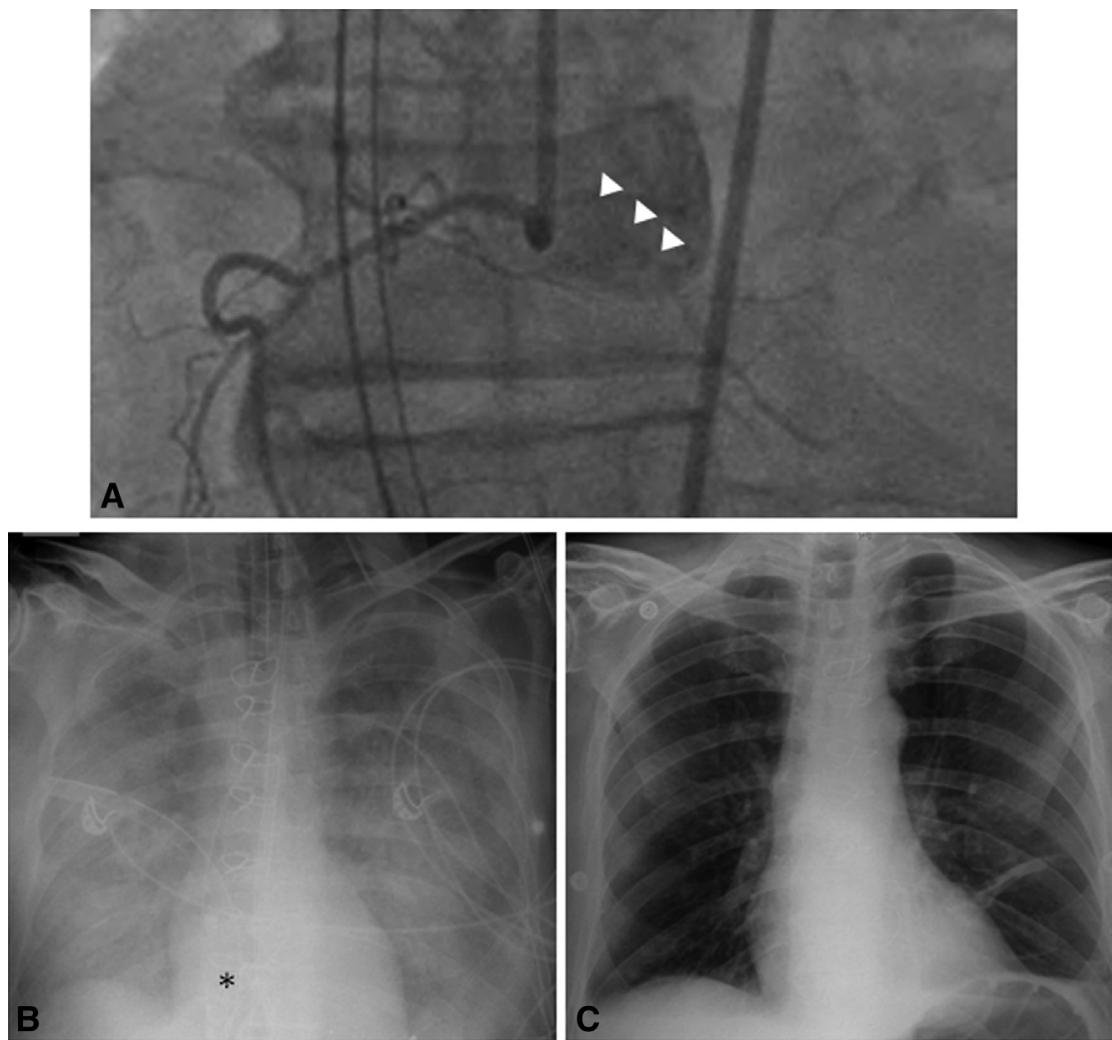

FIGURE 1. A, Right coronary angiogram showing a partial thrombotic occlusion of an ectopic obtuse margin artery (white arrows). The left coronary angiogram was normal. B, Chest radiograph after mitral valve replacement showing pulmonary edema. Note the absence of left atrial dilatation. The venous cannula of extracorporeal life support is indicated by a dark asterisk. $\mathrm{C}$, The chest radiograph was normalized at discharge.

severe mitral regurgitation and a normal left ventricular ejection fraction. An emergency coronary catheterization was performed through the left transfemoral approach, and a partial thrombotic occlusion of an ectopic obtuse marginal artery was diagnosed by right angiography (Figure 1). Left ventriculography confirmed severe mitral
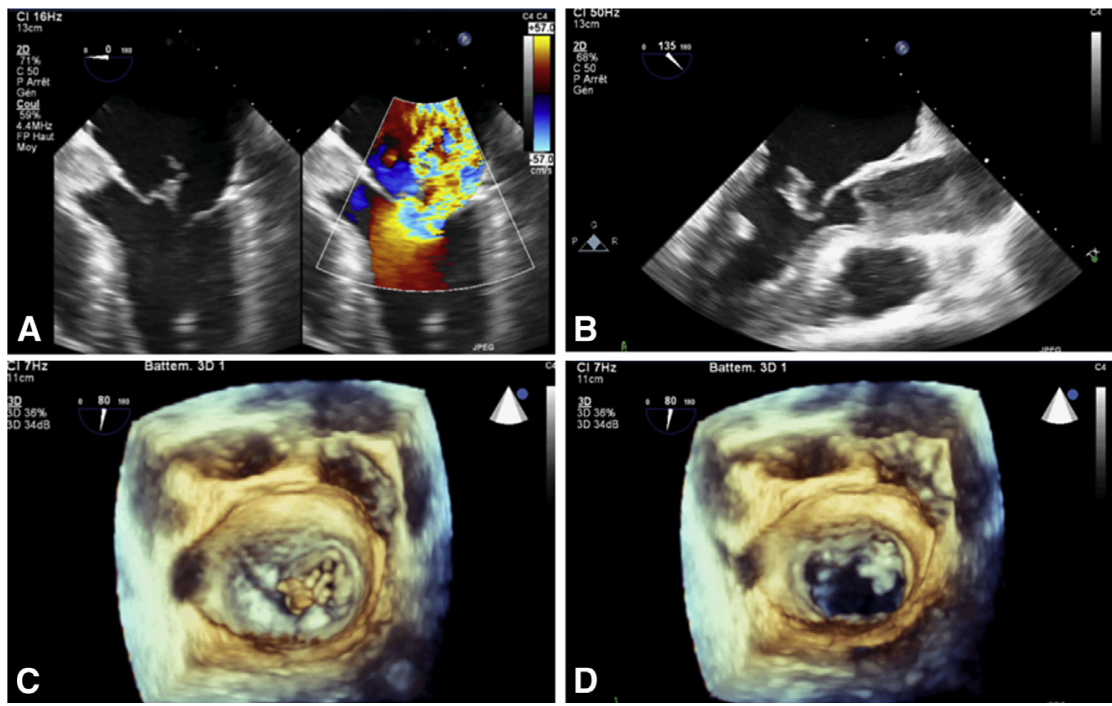

FIGURE 2. A, Two-dimensional transesophageal echocardiographic view with color Doppler in systole shows severe mitral regurgitation secondary to mitral diastasis. B, Two-dimensional transesophageal echocardiographic view in early diastole after implantation of extracorporeal life support shows sludge in the ascending aorta, corresponding to blood stasis in the absence of left ventricular pulsatility. C and D, Three-dimensional transesophageal echocardiographic surgical left atrial view (en face view) shows a rupture of the posterior papillary muscle attached on the ventricular side of A2 during diastole and systole. Note that the motion of the oscillating mass is dependent on the mitral leaflet's motion in contrast to the vegetations. 
regurgitation without ventricular septal rupture. Despite appropriate care, the patient's clinical status continued to worsen, with mean arterial blood pressure dropping below $40 \mathrm{~mm} \mathrm{Hg}$.

Less than 90 minutes after the call to the peripheral hospital, our MRCAU implanted a venoarterial ECMO through a right femoral venoarterial route with percutaneous cannulation. The patient was then brought back by ground to our hospital and immediately transferred to the operating room.

During the intervention, 2-dimensional and real-time 3-dimensional transesophageal echocardiography showed a complete rupture of the posteromedial papillary muscle, resulting in severe mitral regurgitation (Figure 2 and Video 1). Ventricular septal rupture was ruled out with color Doppler echocardiography.

The patient underwent a mitral valve replacement with a bioprosthesis (Medtronic Hancock II Bioprosthetic Valve, $29 \mathrm{~mm}$; Medtronic, Inc, Minneapolis, Minn). The cardiopulmonary bypass duration was 127 minutes, and the aortic crossclamp duration was 63 minutes. The patient required venoarterial ECMO support for 5 days after surgery and needed hemodialysis for 18 days. He was discharged 30 days after the intervention without complication.

\section{DISCUSSION}

Papillary muscle rupture is a rare but often fatal mechanical complication of acute myocardial infarction. It causes acute severe mitral regurgitation, frequently resulting in acute pulmonary edema, cardiogenic shock, or both. Mortality without surgery reaches $80 \%$, and operative mortality within 30 days of surgery ranges from $20 \%$ to $40 \%{ }^{2}$

The development of low cardiac output with a need for ECMO therapy and hemodialysis is associated with the poorest outcome. ECMO must be reserved for carefully selected younger patients with minimal comorbidities, as suggested by Schroeter and colleagues. ${ }^{3}$ Since the creation of our remote cardiac assist unit in 2006, 190 patients have required ECMO placement at the referring hospital and subsequently been shifted to our intensive care unit. Forty-three patients were supported with
ECMO after MRCAU intervention during 2013. Twenty-two of these patients $(51 \%)$ had isolated pulmonary failure and underwent placement of a venovenous bypass, and 21 patients (49\%) had combined cardiopulmonary failure and required venoarterial ECMO. Among these 21 patients, 1 patient $(5 \%)$ underwent transplant, and $10(47 \%)$ were successfully weaned and were alive at hospital discharge.

Indications for venoarterial ECMO were patients in cardiac arrest or cardiocirculatory shock that did not respond to classic maneuvers of resuscitation (systolic blood pressure $<80 \mathrm{~mm} \mathrm{Hg}$ or mean blood pressure $<50 \mathrm{~mm} \mathrm{Hg}$ despite the use of 2 inotropic agents, use of an intra-aortic balloon pump with signs of inadequate perfusion). Our mobile team comprises 1 surgeon, 1 anesthesiologist, and 1 perfusionist and is available on call around the clock. All of the materials for implantation are ready 24 hours a day, 7 days a week. Our miniaturized life support system has a weight of $9400 \mathrm{~kg}$ with autonomous power supply of 180 minutes. These characteristics allow safe interhospital transfer. When ECMO implantation is required outside our institution, road (ambulance) or air (helicopter) transport is used to retrieve the patient as quickly as possible and to return the patient to our intensive care unit.

\section{CONCLUSIONS}

Complete ischemic papillary muscle rupture is a rare but catastrophic complication of acute myocardial infarction. MRCAU allows emergency implantation of ECMO for preoperative stabilization and could be used safely for transportation from a peripheral hospital to a tertiary care cardiac surgery center.

\section{References}

1. Gariboldi V, Grisoli D, Tarmiz A, Jaussaud N, Chalvignac V, Kerbaul F, et al Mobile extracorporeal membrane oxygenation unit expands cardiac assist surgical programs. Ann Thorac Surg. 2010;90:1548-52.

2. Chevalier P, Burri H, Fahrat F, Cucherat M, Jegaden O, Obadia JF, et al. Perioperative outcome and long-term survival of surgery for acute post-infarction mitral regurgitation. Eur J Cardiothorac Surg. 2004;26: $330-5$

3. Schroeter T, Lehmann S, Misfeld M, Borger M, Subramanian S, Mohr FW, et al. Clinical outcome after mitral valve surgery due to ischemic papillary muscle rupture. Ann Thorac Surg. 2013;95:820-4. 